\title{
Effect of FM0807 Inhibits the Proliferation and Migration of RA-FLS through the JAK 2 / STAT3 Signaling Pathway
}

Nanwen Zhang

Fujian Medical University

yize bian ( $\sim$ byz@fjmu.edu.cn )

Fujian Medical University

Ningning Zheng

Fujian Medical University

Liangming Lai

Fujian Medical University

Hongbin Luo

Fujian Medical University

Jianhua Xu

Fujian Medical University

Dunxiong Luo

Fujian Medical University

\section{Xiaole Chen}

Fujian Medical University

\section{Research Article}

Keywords: Rheumatoid arthritis, proliferation, migration, inflammatory, apoptosis

Posted Date: April 6th, 2021

DOI: https://doi.org/10.21203/rs.3.rs-386711/v1

License: (1) (1) This work is licensed under a Creative Commons Attribution 4.0 International License. Read Full License 


\section{Abstract}

Context: It is urgent to develop drugs with independent intellectual property rights and more reasonable price, safe and effective treatment of RA.

Objective: To study the inhibitory effect of curcumin derivative FM0807 on the proliferation of RA-FLS cells and its possible mechanism.

Materials\&Methods: Rheumatiod arthritis fibroblast-like synoviocyte (RA-FLS) were cultured in alone or in the presence of FM0807 $(25 \mu \mathrm{M}, 50 \mu \mathrm{M}$ and $100 \mu \mathrm{M})$. MTT method was used to analyze the effect of cell proliferation, Hoechst 33342/PI staining was used to observe the effect of apoptosis. Cell migration assay was used to observe the effect of migration ability of RA-FLS. The expression of JAK2,STAT3, IL- 6 and IL-1 $\beta$ gene was determined by RT-PCR method. Western blotting assay was used to detect the expression of JAK2-STAT3 signaling pathway related proteins.

Results: A 72-hour exposure FM0807 result in cell proliferation inhibition significantly $\left(\mathrm{IC}_{50}=41.38 \mu \mathrm{M}\right)$. The migration of RA-FLS is inhibited by FM0807 $100 \mu \mathrm{M}$ in $72 \mathrm{~h}(P<0$ 05). High Content Screening showed that the apoptosis rate of RA-FLS was up regulated after treated FM0807. Presence of FM0807 in the culture medium strongly inhibited JAK2,STAT3,IL-6 and IL-13mRNA expression in a concentrationdependent manner. Western blot demonstrated a substantially reduced protein expression of phosphorylation of JAK2 and STAT3. At the same time, the anti-apoptotic protein Bcl-2 and the key proteins of PI3K cell pathway including Akt and mTOR were down-regulated. on the contrary, the expression of apoptosis execution protein Cleaved-caspase3 and Bax were up-regulated.

Conclusion: Curcumin derivative FM0807 may inhibit the proliferation of RA-FLS and promote its apoptosis by inhibiting JAK2-STAT3 signaling pathway.

\section{Introduction}

The typical pathological changes of Rheumatoid arthritis(RA) are synovitis, the main characteristics of which are the decrease of synovial cell apoptosis, tumor-like growth and the secretion of inflammatory factors $^{[1]}$. The healthy synovium has an intimal lining that contains macrophage-like synovial cells and fibroblasts-like synovial cells (Fibroblast-like synoviocytes, FLS) and subintimal lining, adipocytes, blood vessels, and dispersed immune cells containing fibroblasts. However, the RA-FLS of fibroblasts-like synovial cells in RA patients has the characteristics of excessive activation and decreased apoptosis. At the same time, the signal transduction network involved in the inflammatory process of RA is very complex. Although it has been gradually uncovered, it has not been fully clarified. Pro-inflammatory cytokines, such as IL-6, IL-8, COX-2 and so on, induce different intracellular transduction events and ultimately regulate the production of a series of cytokines ${ }^{[2]}$. It has been confirmed that various pathogenic signal transduction pathways in RA are nuclear factor B (NF-kB), mitogen-activated protein kinase. (MAPK), spleen tyrosine kinase (SYK), phosphoinositol 3-kinase (PI3K), Interleukin(IL)-17 and Loading [MathJax]/jax/output/CommonHTML/jax.js of transcription(JAK-STAT). 
With Pfizer's oral JAK inhibitor Xeljanz (tofacitinib, tofatinib) approved by Japanese PMDA in March 2013 , methotrexate alone or in combination with methotrexate for the treatment of rheumatoid arthritis (RA) ${ }^{[3]}$, in recent years, many international pharmaceutical companies have devoted themselves to the research and development of JAK inhibitors. At present, the first generation of JAK inhibitor: Ruxolitinib (Rosotinib, Novartis), Tofacitinib and Baricitinib (Barrick Tinib, Lilai), which are characterized by the ability to inhibit multiple JAK subtypes of kinases at the same time ${ }^{[4]}$. Second generation JAK inhibitors: including Filgotinib, Upadacitinib (Upatinib, Abervi) and Solcitinib selectively inhibit JAK1; Decermotinib and PF06651600 selectively inhibit JAK3 ${ }^{[5]}$; The selective inhibition of tyrosine kinase (Tyk)2, by BMS986165,NDI021232, NDI031407, PF06700841 and SAR20347 is characterized by selective JAK inhibitors designed for different subtypes, among which there are many small molecular compounds with RA as indication.

JAK-STAT signal transduction, originally identified as interferon signal transduction pathway, mediates the immune response of various cytokines and the effects of several growth factors and hormones, and is involved in inflammation and cancer processes. Various strains of JAK and STAT molecules mediate different signals, which leads to different functions. The binding of ligands to their receptors induced the assembly of active receptor complexes and the subsequent phosphorylation of JAK (JAK1,JAK2 and JAK3) and Tyk2 associated with receptors. Phosphorylation activates JAK receptor subunits, including other substrates, and provides docking sites for STAT phosphorylation. The phosphorylated STAT is released from the receptor complex and forms homologous or heterodimer, which is then transferred to the nucleus and binds to the promoter region of the specific target gene, thus regulating the transcription of inflammation-related genes ${ }^{[6]}$. The continuous activation of JAK/STAT signal transduction in synovial joints of RA patients leads to the increase of matrix metalloprotease gene expression and the frequency of apoptotic cartilage cells, and the most prominent problem in inflamed synovial tissues is "apoptosis resistance". Small molecule JAK inhibitors, represented by topatinib, have a good effect on improving $\mathrm{RA}^{[7]}$, but these drugs are more expensive and many patients are deterred from doing so. The New England Journal reports that patients with RA who have been treated with Barretinib for a long time, in addition to gastrointestinal reactions, also increase the risk of infection, such as herpes zoster infection, resulting in a decrease in high density lipoprotein, increased serum creatinine and transaminase, and so on ${ }^{[8]}$. Therefore, it is urgent to develop drugs with independent intellectual property rights and more reasonable price, safe and effective treatment of RA, which is in line with the national conditions of our country.

Curcumin is a polyphenolic compound isolated from the roots of Curcuma longa, a plant. It is traditionally used for pain and wound healing. Recent studies have shown that curcumin can improve multiple sclerosis, rheumatoid arthritis, psoriasis and inflammatory bowel disease in human or animal models. Curcumin inhibits these autoimmune diseases by regulating inflammatory cytokines such as IL1, IL-6, IL-12, TNF-a, IFN-ya and related JAK-STAT, AP-1 and NF-KB signal transduction pathways in immune cells ${ }^{[9]}$.Curcumin can reduce the expression of HMGB1 in brain tissue after cerebral ischemia 
signaling pathway ${ }^{[10]}$. It can increase cell adhesion by down-regulating the expression of total proteins of p-JAK, p-STAT3 and STAT3 ${ }^{[11]}$, and inhibit the migration and invasion of melanoma cells and enhance the apoptosis of these cells by regulating multiple pathways, including JAK2/STAT3 ${ }^{[12,13]}$. It may provide a theoretical basis for the clinical treatment of RA by weakening the phosphorylation of Jak2, downregulating the transcriptional activities of STAT1 and STAT3 DNA, and inhibiting the proliferation of RA$\mathrm{FLS}^{[14]}$.

Previous experiments have confirmed that curcumin derivative FM0807 used in experiment has high safety and good anti-arthritis activity in vivo ${ }^{[15]}$.FM0807 can inhibit the release of inflammatory factors from RAW264.7 cells induced by LPS, and it is more effective on providing protection than curcumin,a equimolar positive control drug ${ }^{[16]}$.It is speculated that FM0807 may have anti-inflammatory effect mediated by JAK2-STAT3 signal pathway, which is similar to curcumin. It is worth further confirming. The purpose of this chapter is to study the effects of FM0807 on the proliferation, apoptosis and potential molecular mechanism of RA-FLS, in order to elucidate the mechanism and possible targets of antiexperimental arthritis.

\section{Materials And Methods}

\subsection{The culture of RA-FLS cells}

After digestion, RA-FLS (Shanghai institute of cell biology, Chinese academy of sciences,China) were washed extensively and cultured in serum-free Dulbecco's modified Eagle's medium (DMEM)/F12 (Gibco,USA). After overnight culture, nonadherent cells were removed, and adherent cells were re-cultured in DMEM/F12 supplemented with $10 \%$ fetal bovine serum (Gibco,USA), $100 \mathrm{U} / \mathrm{ml}$ penicillin, and 100 $\mu \mathrm{g} / \mathrm{ml}$ streptomycin in a humidified $37^{\circ} \mathrm{C}$ and $5 \% \mathrm{CO}_{2}$ incubator (Thermo Foma, USA) ${ }^{[17]}$. Subculturing was performed when cells reached $80-90 \%$ confluence. The culture medium was replaced once the next day and continued to grow.

\subsection{MTT assay}

MTT assay is a method to detect cell survival and growth. Cells in logarithmic growth phase were inoculated into 96 well plates with $2 \times 10^{3} \mathrm{~mL}^{-1}$ cells, $180 \mu \mathrm{L}$ per hole. $5 \% \mathrm{CO}_{2}$ and $37^{\circ} \mathrm{C}$ for 24 hours were used when the cells were laid to $70-80 \%$ flat bottoms. The experimental group was treated with FM0807 of different concentration, while the control group was treated with the same amount of complete medium. Each group had at least three compound pores. After $24 \mathrm{~h}, 48 \mathrm{~h}$ and $72 \mathrm{~h}$ of continuous culture at $37{ }^{\circ} \mathrm{C}$, the MTT solution of $5 \mathrm{mg} \cdot \mathrm{mL}^{-1}$ was added to each pore for $20 \mu \mathrm{L} /$ pore. After $4 \mathrm{~h}$ of continuous culture, the supernatant was discarded by centrifugation, $150 \mu \mathrm{L}$ DMSO was added ${ }^{[18]}$, the Microoscillator was added for $10 \mathrm{~min}$, and the absorbance (OD value) was immediately measured at $570 \mathrm{~nm}$ wavelength by enzyme-labeling instrument.Cell growth inhibition rate and $\mathrm{IC}_{50}$ were calculated according to absorbance. The inhibition rate of cell growth $(\%)=($ control group OD value - experimental group OD 


\subsection{Grouping of RA-FLS cell experiments}

According to the results of 2.2, the experiment was divided into four groups: control group, FM0807 $25 \mu \mathrm{M}$

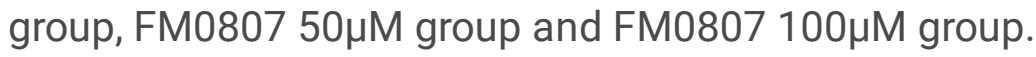

\subsection{Hoechst 33342/PI staining}

The apoptosis of cells was evaluated using a Hoechst 33342 kit (Solarbio, Beijing, China) according to manufacturer's instructions. RA-FLS was seeded into 24-well plates, and treated with various concentrations of FM0807. In brief, following the treatment for 72 hours, the cells were stained with Hoechst 33342 and PI at $4^{\circ} \mathrm{C}$ for $20 \mathrm{~min}$, washed twice with PBS. The High Content Screening (Thermo scientific, USA) was used to capture and analyze the images. Blue-labeled cells are total cells, Red-labeled cells are dead cells, or necrotic cells or late apoptotic cells ${ }^{[19]}$.

\subsection{Cell Scratch Test}

The spreading and migration capabilities of RA-FLS cells were assessed using a scratch wound assay which measures the expansion of a cell population on surfaces ${ }^{[20]}$. The cells were seeded into 6 -well tissue culture dishes containing pre-coated coverslips.

A linear wound was generated in the monolayer with a sterile $100 \mu l$ plastic pipette tip. Any cellular debris was removed by washing the coverslips with phosphate buffer saline (PBS). Then the cells were treated with $0 \mu \mathrm{M}, 25 \mu \mathrm{M}, 50 \mu \mathrm{M}$ and $100 \mu \mathrm{M}$ FM0807 for 72 hours, and no additives were used as internal controls. Finally, the cells were fixed with $4 \%$ paraformaldehyde for $15 \mathrm{~min}$ and observed under microscope and measured with Image pro plus software. Three representative images from each coverslip of the scratched areas under each condition were photographed to estimate the relative migration cells for quantifications.

\subsection{Transwell lab experiment}

The grouping method is the same as 2.5. After FM0807 interfered with RA-FLS for 72 hours, DMEM serum-free medium was used to prepare single cell suspension. Cell counts were made and cell density was adjusted to $6 \times 10^{4} \mathrm{~mL}^{-1}$.In Transwell chamber, the $600 \mu \mathrm{L}$ DMEM medium containing $10 \%$ FBS was added to the basement of the chamber, and then gently placed into the upper chamber to avoid the formation of bubbles. Cell suspension of $200 \mu \mathrm{L}$ was added to the upper chamber of Transwell chamber and incubated for 24 hours in a cell culture box containing $5 \% \mathrm{CO}_{2}$ at $37{ }^{\circ} \mathrm{C}$. Fixation and dyeing: The upper chamber moves into the blank hole, the cotton swab wipes the inner surface of the membrane, removes the unmigrated cells, washes the PBS buffer three times, fixes 30 mins with appropriate amount of methanol, then changes to other holes, and stains with crystal violet for 30 mins.After dyeing, PBS was cleaned three times, the background was removed, and then observed, photographed and counted under the microscope.Migration inhibition rate was calculated by the following formula: 
where $\mathrm{NO}$ and $\mathrm{N} 1$ are average numbers of migrated cells in drug group and control group

\subsection{Relative Quantitative Real-Time PCR}

Cells treated with $0 \mu \mathrm{M}, 25 \mu \mathrm{M}, 50 \mu \mathrm{M}$ and $100 \mu \mathrm{M}$ FM0807 for72h,total RNA was isolated from the RA-FLS using Micro total RNA extraction kit (TIANMO Biotech, Beijng,China). Total RNA was isolated from the RAFLS using TRIzol reagent (Gibco BRL, Grand Island, NY, USA). The complementary DNA was synthesized and amplified by real-time PCR using a Prime Script RT Reagent Kit (Takara). Real-time PCR reactions were performed in a Mini Option TM RT-PCR system (Bio-Rad, Hercules, CA, USA) using SYBR Premix Ex Taq II (FINNZYMES, Espoo, Finland). The real-time-PCR conditions included $95^{\circ} \mathrm{C}$ for $10 \mathrm{~min}$, followed by 40 cycles of $95^{\circ} \mathrm{C}$ for $15 \mathrm{~s}$ and $60^{\circ} \mathrm{C}$ for $31 \mathrm{~s}$. Primer specificity was monitored using product melting curves in each reaction well. Raw data were normalized and expressed relative to the housekeeping gene $\mathrm{GAPDH}$ as $2^{-\triangle \Delta C t}$ values. The relative amplification efficiencies of the primers were tested and shown to be similar ${ }^{[22]}$.

The following primers were used:

(1) IL-6 primer sequences were forward 5- $G \forall C T \mathbb{C}$ T $C T \mathbb{C} A \mathbb{C} G C G$ - 3 and reverse 5 - $C T G \forall$ GAGGTGAGTGGCTGTC - 3.

(2) IL-1 $\beta$ primer sequences were forward 5- $C A G \forall G T A \mathbb{C} T G A G C T C G \mathbb{C}$ - 3 and reverse 5 - AGA T CGTAGCTGGATGC $G$ - 3.

(3) JAK2 primer sequences were forward 5- GCTGAG T CG $\forall$ GCTAGCAGG - 3 and reverse 5 - $G \forall G \mathbb{C} C G T C A C A G$ T GTCT - 3.

(4) STAT3 primer sequences were forward 5-ACAGGATGGC $C \forall T G G \forall T$ - 3 and reverse 5 - CTC T GCAGG $\forall$ GCGGCTAT - 3.

(5) GAPDH primer sequences were forward 5- $G \forall A T G \forall T G G G C A G \mathbb{C} G \mathrm{~T}-3$ and reverse 5 - $A T C A \mathbb{C} C G G A G G A G \forall A T C G$ - 3. Primers were synthesized by Sangon Biotech (Shanghai,China).Data were analyzed with the delta delta Ct method.

\subsection{Western blotting assay}

Cells in the logarithmic growth phase in each group were harvested in precooled 3 to 5 times volume lysis buffer composed of $1 \mathrm{M}$ Tris- $\mathrm{HCl}$ pH 8.0, $5 \mathrm{M} \mathrm{NaCl}, 10 \%$ Nonidet P40, and one tablet of protease inhibitor cocktail (Roche, Indianapolis, IN, USA). Cells were then incubated on ice for 10 minutes and centrifuged at 12,000 rpm for 10 minutes at $4^{\circ} \mathrm{C}$. The pellet was discarded and the total protein concentration in the supernatant was determined using the Bio-Rad protein assay kit (Bio-Rad, Hercules, CA, USA). Proteins $(50 \mu \mathrm{g})$ were separated by $10 \%$ SDS-PAGE gel electrophoresis, transferred to PVDF membranes (Roche Switzerland), and probed with appropriate antibodies. Antibodies to $\beta$ tubelin(Cat.\#2146),JAK2(Cat.\#D2E12),p-STAT3(Y705,Cat.\#9134),STAT3 
(Cat.\#12640),p-JNK(Tyr183/185,Cat.\#9251),JNK(Cat.\#6318),Akt(Cat.\#9272)and pmTOR(Ser2448,Cat.\#6318) were obtained from Cell Signaling Technology (Beverly, MA, USA). Antibodies to p-JAK2(Tyr1007/1008,Cat.\#ab32101),Cleaved caspase-3 (Cat.\#ab184787), Bax(Cat.\#ab32503) and Bcl-2 $\square$ Cat.\#ab182858』were obtained from Abcam (Cambridge, UK). Primary antibodies(all 1:1000) were incubated overnight at $4^{\circ} \mathrm{C}$ and horseradish peroxidase conjugated secondary antibodies were incubated for 1 hour at room temperature ${ }^{[23]}$. Proteins were detected with the ECL chemiluminescence hypersensitive color development kit (Cat: 36208 es76, LOT: S8828040,Yeasen biological technology, Shanghai, China). Densitometry values were analyzed and quantified with Image J Software.

\subsection{Statistical analysis}

Data are expressed as the mean \pm standard deviation of three independent experiments. Statistical results were analyzed using the Mann-Whitney test. Data were analyzed using SPSS version 19.0 for Windows (SPSS Inc.,Chicago, IL, USA). P-values less than 0.05 were considered statistically significant.

\section{Result}

\subsection{Effect of FM0807 on RA-FLS proliferation}

MTT assay was used to analyze the effect of FM0807 on the proliferation of RA-FLS cells, and the experimental concentration of FM0807 was preliminarily determined according to the preliminary experimental results. The results showed that FM0807 with $25 \mu \mathrm{M}, 50 \mu \mathrm{M}$ and $100 \mu \mathrm{M}$ interfered with RAFLS for 72 hours. With the increase of the concentration of the drug, the cells gradually changed from spindle-shaped to slender, irregular and round, and eventually led to cell loss of growth ability(Fig. 1A). The inhibitory rates were $(29.64 \pm 2.55) \%,(46.02 \pm 4.70) \%$ and $(61.10 \pm 3.27) \%$ respectively, which were significantly different from those in the control group $(P<0.001)$. The $\mathrm{IC}_{50}$ of FM0807 treated RA-FLS for 72 hours was calculated by GraphPad Prism 8.0 software was $41.38 \mu \mathrm{M}$. The $95 \%$ confidence interval was $(28.48,60.12) \mu \mathrm{M}$ (Fig. 1B).

\subsection{Effect of FM0807 on RA-FLS death}

In order to observe the death effect of curcumin derivative FM0807 on RA-FLS (Fig. 1B), PI/Hoechst 33342 double staining method was used to observe the death of RA-FLS in gradient concentration FM0807. Fluorescence microscopy showed that the control group showed uniform blue fluorescence. Curcumin derivative FM0807 at concentrations of $25 \mu \mathrm{M}, 50 \mu \mathrm{M}$ and $100 \mu \mathrm{M}$ could effectively promote cell death for 72 hours(Fig. 1C). The results of high connotation analysis showed that the mortality rate of control group was $8.72 \pm 1.61 \%$. Compared with control group, the mortality rate of FM0807 group $(25 \mu \mathrm{M}$, $50 \mu \mathrm{M}, 100 \mu \mathrm{M})$ was $(62.53 \pm 27.32) \%(P<0.01),(86.64 \pm 9.09) \%(P<0.001)$ and $(95.31 \pm 2.15) \%(P<$ 0.001), (Fig. 1D). These results suggest that FM0807 can induce RA-FLS death, and increase the death rate of RA-FLS in a dose-dependent manner.

\subsection{Effect of FM0807 on the migration ability of RA-FLS in}


The effects of curcumin derivative FM0807 on the migration ability of RA-FLS in vitro were examined by scratch and migration experiments. The scratch test showed that the migration ability of each FM0807 dose group decreased significantly after 72 hours compared with the control group (all $P<0.05$ ), and was dose-dependent from the Fig. 2A and 2B. According to the number of cells per field of view, it can be seen from the chamber experiments in Fig. $2 \mathrm{C}$ that the control group, FM0807 $25 \mu \mathrm{M}, 50 \mu \mathrm{M}$ and $100 \mu \mathrm{M}$ acting the results were $(229.33 \pm 33.34),(205.22 \pm 14.25),(165.00 \pm 38.94)$ and $(103.22 \pm 17.07, P<0.001)$ for $72 \mathrm{~h}$ in Fig. 2D.

\subsection{Effect of FM0807 on the expression levels of JAK2- STAT3 signaling pathway in RA-FLS}

RT-qPCR showed that FM0807 significantly changed the expression levels of JAK2,STAT3,IL-6 and IL$1 \beta m R N A$ in RA-FLS $(P<0.001)$ in a dose-dependent manner. The results of Fig. 3A-D showed that the

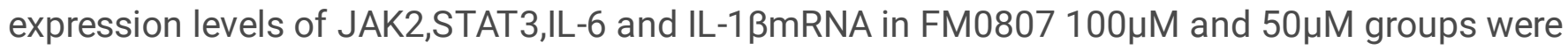
significantly lower than those in the blank group $(P<0.05)$. The expression level of IL-1 $\beta m R N A$ in low dose group $(25 \mu \mathrm{M})$ was also significantly decreased $(P<0.001)$.

In order to further explore the molecular mechanism of curcumin derivative FM0807 inhibiting the proliferation, migration and inflammatory response of RA-FLS cells, we focused on the effect of FM0807 on the activation of JAK2-STAT3 pathway. The expression levels of p-JAK2/JAK2,p-STAT3/STAT3,Akt,pmTOR,Cleaved caspase-3,

Bax and Bcl-2 protein in RA-FLS were detected by Western blotting. Western blot results showed that FM0807 $50 \mu \mathrm{M}$ and $100 \mu \mathrm{M}$ could significantly inhibit the phosphorylation of JAK2 protein $(P<0.05$ or $P<$ $0.001)$ and then inhibit the activation of STAT3 $(P<0.05$ or $P<0.01)$. At the same time, FM0807 50 $\mu \mathrm{M}$ and $100 \mu \mathrm{M}$ could also down-regulate the key proteins Akt and mTOR of PI3K signal pathway $(P<0.001$ or $P<0.01)$, and ultimately lead to the decrease of the expression of anti-apoptotic protein Bcl-2 $(P<$ 0.001). On the contrary, the expression of Bax and Cleaved caspase3 were significantly up-regulated (all $P<0.001$ ) (Fig. 3E-F).

\section{Discussion}

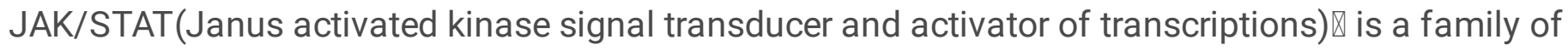
important intracellular signal transduction pathways discovered in recent years and is also one of the important pathways that mediate inflammatory responses. The binding of cytokines to the JAKs receptor causes dimerization of the receptor molecule, causing tyrosine phosphorylation of the JAKs coupled to the receptor, and the activated JAKs catalyze the phosphorylation of the tyrosine residues of the receptor itself. The corresponding STATs docking sites are formed, allowing STATs to bind to the receptor via the SH2 domain, and phosphorylate them under the action of JAKs, then enter the nucleus and activate the 
corresponding gene transcription and expression, such as Bcl-2 levels were increased, whereas Bax and caspase-3 levels were decreased ${ }^{[24]}$.

Rheumatoid arthritis (RA) is a chronic autoimmune disease characterized by synovial hyperplasia, chronic inflammation, and progressive destruction of the joints.. Many inflammatory mediators such as

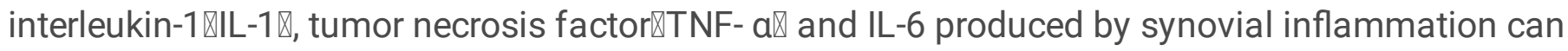
mediate the production of proteases such as MMP-1, 3 and 13 that degrade cartilage matrix by chondrocytes, thus aggravating the degeneration process of articular cartilage ${ }^{[25]}$. IL-6 signal transduction is initiated by binding with IL-6R complex containing signal transduction protein 130 . JAK is activated by phosphorylation of GP 130, which promotes the recruitment of STAT family members, such as STAT 3. JAK-mediated phosphorylation of STAT 3 is essential for its nuclear translocation and transcription of downstream target genes. STAT3 is closely related to osteoclast formation. Studies have shown that the expression of RANKL mediated by IL-6/sIL-6R complex requires STAT3 activation ${ }^{[26]}$. Activated JAK2 also activates PI3K/Akt pathway and promotes proliferation and survival of MAPK pathway ${ }^{[27]}$.

Rheumatiod arthritis fibroblast-like synoviocyte (RA-FLS, also known as type B synovial cells) are a special kind of mesenchymal derived cells. RA-FLS is the main cell component to maintain the dynamic balance of synovium. In the context of RA, RA-FLS promotes the progress of synovial proliferation and inflammation. New evidence suggests that inhibiting RA-FLS effector molecules may be beneficial ${ }^{[28]}$. In the results of this part, the positive PI of RA-FLS cells may be necrosis or late apoptosis, indicating that FM0807 may have the effect of inducing apoptosis. At the same time, in immunoelectrophoresis experiments, FM0807 can significantly inhibit the activation of JAK2 protein, and also down-regulate the expression of key proteins Akt and p-mTOR of PI3K/Akt pathway, inhibit the expression of anti-apoptotic protein $\mathrm{Bcl}-2$, and increase the expression of Bax and Cleaved caspase3, thereby exerting a role in inducing apoptosis of RA-FLS. This study shows that the curcumin derivative FM0807 can inhibit the proliferation of RA-FLS, reduce cell migration and promote apoptosis.

Inhibition of JAK/STAT activity can induce apoptotic response ${ }^{[29]}$. It has been confirmed that RA-FLS in the intimal lining of RA expresses high levels of STAT $1^{[30]}$, and that the expression of STAT3 increases in the synovial lining of RA and in the experimental arthritis model ${ }^{[31]}$. Continuous activation of STAT3 contributes to the expression of anti-apoptotic molecules, while blockade of STAT3 promotes the apoptosis of RA-FLS ${ }^{[32]}$. Previous studies have shown that JAK2/STAT 1/3 signaling pathway may be the upstream mechanism controlling the spread of RA-FLS ${ }^{[33]}$. Studies have shown that matrine inhibits the proliferation of human cholangiocarcinoma cells and induces apoptosis of JAK2/STAT 3 signal transduction ${ }^{[34]}$. The appearance of RA-FLS with tumor-like invasive phenotype may also justify the use of cancer therapy for RA ${ }^{[35]}$. It found that curcumin derivative FM0807 can inhibit JAK2 phosphorylation, suggesting that curcumin derivative FM0807 may induce apoptosis and migration by inhibiting JAK2/STAT3 signal. 
In conclusion, the results of this study suggest that curcumin derivative FM0807 has a strong antiproliferation and death (necrosis or late apoptosis) effect on RA-FLS, which may be related to antiJAK2/STAT3 signal activation. However, how the drug regulates JAK2 expression and the specific target of action need to be clarified by further experiments.

\section{Declarations}

\section{ACKNOWLEDGEMENTS}

The authors wish to thank the Fujian Medical University for providing their equipment to conduct the experiment.

\section{AUTHOR CONTRIBUTION}

N.Z. and X.C. designed the study. Y.B., N.Z., L.L. and H.L. performed the experiments, analyzed the data and wrote the draft. N.Z. and X.C. finalized the manuscript. X.L. contributed analysis tools. The first corresponding author is Xiaole Chen

\section{FUNDING}

This work was supported by Fujian provincial health technology project\China (Grant No. 2018-ZQN-61, 2018-ZQN-64) , Natural Science Foundation of Fujian Province, China (Grant No. 2019J01306, 2020J01634), The Emergency Project on New Coronavirus Pneumonia Prevention Research of Fujian Medical University (Grant No. 2020YJ004) and the Joint Funds for the Innovation of Science and Technology, Fujian Province [Grant No. 2019Y9111].

\section{DATA AVAILABILITY}

The datasets used and/or analyzed during the current study are available from the corresponding author on reasonable request.

\section{Consent for Publication.}

The manuscript is approved by all authors for publication.

\section{Competing Interests.}

The authors declare that they have no conflict of interest. 


\section{References}

1. L. XZ et al., 2018 Effect of Dishevelled 2 on apoptosis in rheumatoid arthritis fibroblast-like synoviocytes through inhibiting JAK-STAT pathway. Zhonghua yi xue za zhi 98, 1189-1193.

2. K. B, B. T, D. E, K. H, S. G, 2013 Anti-inflammatory and apoptotic effects of the polyphenol curcumin on human fibroblast-like synoviocytes. International immunopharmacology 15, 400-405.

3. F. R, 2018 Tofacitinib in the treatment of active rheumatoid arthritis in adults. Immunotherapy 10, 3956.

4. B. M, T. B. PA, 2012 JAK2 Inhibition: Reviewing a New Therapeutical Option in Myeloproliferative Neoplasms. Advances in hematology 2012, 535709.

5. N. P, 2014 Selective JAK inhibitors in development for rheumatoid arthritis. Expert opinion on investigational drugs 23, 1067-1077.

6. S. L, E. CJ, 2019 Upadacitinib for the treatment of rheumatoid arthritis. Expert review of clinical immunology 15, 13-25.

7. M. CJ, 2018 The role of the JAK/STAT signal pathway in rheumatoid arthritis. Therapeutic advances in musculoskeletal disease 10, 117-127.

8. T. PC et al., 2017 Baricitinib versus Placebo or Adalimumab in Rheumatoid Arthritis. The New England journal of medicine 376, 652-662.

9. B. JJ, 2007 Curcumin and autoimmune disease. Advances in experimental medicine biology 595, 425-451.

10. Y. Wu et al., 2018 Study on the mechanism of JAK2/STAT3 signaling pathway-mediated inflammatory reaction after cerebral ischemia. Molecular Medicine Reports 17.

11. Z. BZ, L. TD, C. G, Z. JX, K. X, 2018 The effect of curcumin on cell adhesion of human esophageal cancer cell. European review for medical pharmacological sciences 22, 551-560.

12. C.-G. A et al., 2019 Curcumin prevented human autocrine growth hormone (GH) signaling mediated NF-KB activation and miR-183-96-182 cluster stimulated epithelial mesenchymal transition in T47D breast cancer cells. Molecular biology reports 46, 355-369.

13. S. A, L. YS, 2013 Molecular mechanisms of curcumin action: signal transduction. BioFactors 39, 2736.

14. W. H et al., 2012 Inhibitory Effect of Curcumol on Jak2-STAT Signal Pathway Molecules of Fibroblast-Like Synoviocytes in Patients with Rheumatoid Arthritis. Evidence-based complementary alternative medicine : eCAM 2012, 746426.

15. Z. N et al., 2019 FM0807 decelerates experimental arthritis progression by inhibiting inflammatory responses and joint destruction via modulating NF-KB and MAPK pathways. Bioscience reports 39.

16. W. Y et al., 2018 Effects of FM0807, a novel curcumin derivative, on lipopolysaccharide-induced inflammatory factor release via the ROS/JNK/p53 pathway in RAW264.7 cells. Bioscience reports 38. 
17. N. HQ et al., 2018 Combination of 4-hydroperoxy cyclophosphamide and methotrexate inhibits IL6/sIL-6R-induced RANKL expression in fibroblast-like synoviocytes via suppression of the JAK2/STAT3 and p38MAPK signaling pathway. International immunopharmacology 61, 45-53.

18. Z. T et al., 2016 p53 predominantly regulates IL-6 production and suppresses synovial inflammation in fibroblast-like synoviocytes and adjuvant-induced arthritis. Arthritis research therapy 18, 271.

19. K. M. Hanson, J. N. Finkelstein, 2019 An accessible and high-throughput strategy of continuously monitoring apoptosis by fluorescent detection of caspase activation. Analytical Biochemistry.

20. M. XG, Y. SW, 2014 Dexamethasone disrupts cytoskeleton organization and migration of T47D Human breast cancer cells by modulating the AKT/mTOR/RhoA pathway. Asian Pacific journal of cancer prevention : APJCP 15, 10245-10250.

21. W. J et al., 2019 Effect of polysaccharide from Undaria pinnatifida on proliferation, migration and apoptosis of breast cancer cell MCF7. International journal of biological macromolecules 121, 734742.

22. Q. K et al., 2018 Methotrexate Combined with 4-Hydroperoxycyclophosphamide Downregulates Multidrug-Resistance P-Glycoprotein Expression Induced by Methotrexate in Rheumatoid Arthritis Fibroblast-Like Synoviocytes via the JAK2/STAT3 Pathway. Journal of immunology research 2018, 3619320.

23. C. JY, P. KY, P. SH, L. SI, K. SK, 2013 Regulatory effect of calcineurin inhibitor, tacrolimus, on IL-6/sIL6R-mediated RANKL expression through JAK2-STAT3-SOCS3 signaling pathway in fibroblast-like synoviocytes. Arthritis research therapy 15, R26.

24. Y. Y, D. Q, L. R, 2017 Matrine induces the apoptosis of fibroblast-like synoviocytes derived from rats with collagen-induced arthritis by suppressing the activation of the JAK/STAT signaling pathway. International journal of molecular medicine 39, 307-316.

25. R. $\mathrm{N}$ et al., 2005 Association of the risk of osteoarthritis with high innate production of interleukin1 beta and low innate production of interleukin-10 ex vivo, upon lipopolysaccharide stimulation. Arthritis rheumatism 52, 1443-1450.

26. N. Choudhary, L. K. Bhatt, K. S. Prabhavalkar, 2018 Experimental animal models for rheumatoid arthritis. Immunopharmacol Immunotoxicol 40, 1-8.

27. M. SC, L. RL, 2014 Molecular pathways: molecular basis for sensitivity and resistance to JAK kinase inhibitors. Clinical cancer research : an official journal of the American Association for Cancer Research 20, 2051-2059.

28. G. R, R. M, 2017 Fibroblast-like synoviocytes-dependent effector molecules as a critical mediator for rheumatoid arthritis: Current status and future directions. International reviews of immunology 36 , 20-30.

29. Y. CT et al., 2013 A sesquiterpene lactone antrocin from Antrodia camphorata negatively modulates JAK2/STAT3 signaling via microRNA let-7c and induces apoptosis in lung cancer cells. Carcinogenesis 34, 2918-2928. 
30. K. PV et al., 2004 Activation of the STAT1 pathway in rheumatoid arthritis. Annals of the rheumatic diseases 63, 233-239.

31. S. T et al., 2001 Induction of the cytokine signal regulator SOCS3/CIS3 as a therapeutic strategy for treating inflammatory arthritis. The Journal of clinical investigation 108, 1781-1788.

32. K. SK et al., 2011 Melittin enhances apoptosis through suppression of IL-6/sIL-6R complex-induced NF-KB and STAT3 activation and Bcl-2 expression for human fibroblast-like synoviocytes in rheumatoid arthritis. Joint, bone, spine : revue du rhumatisme 78, 471-477.

33. P. JS et al., 2014 JAK2-STAT3 blockade by AG490 suppresses autoimmune arthritis in mice via reciprocal regulation of regulatory T Cells and Th17 cells. Journal of immunology research 192, 4417-4424.

34. Y. N et al., 2015 Matrine suppresses proliferation and induces apoptosis in human cholangiocarcinoma cells through suppression of JAK2/STAT3 signaling. Pharmacological reports : PR 67, 388-393.

35. L. GF, Q. YH, D. PQ, 2015 Andrographolide inhibits the migration, invasion and matrix metalloproteinase expression of rheumatoid arthritis fibroblast-like synoviocytes via inhibition of HIF1a signaling. Life sciences 136, 67-72.

\section{Figures}

Fig 1

A

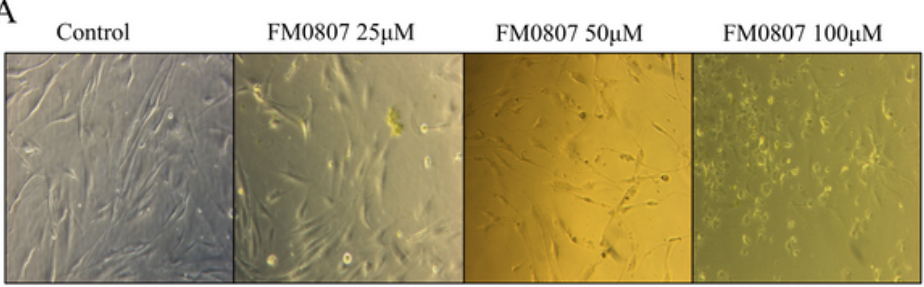

B

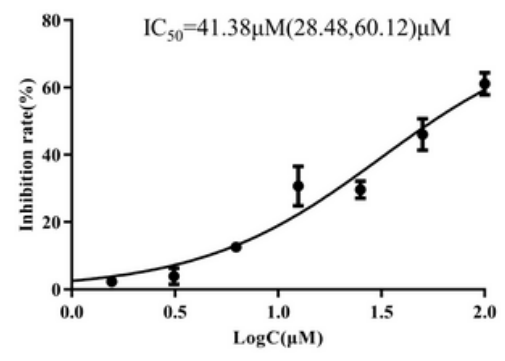

C

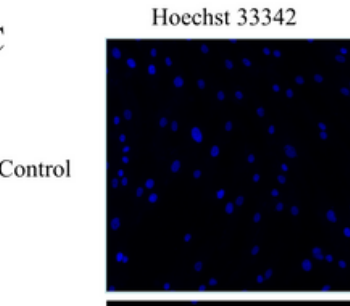

FM0807 $25 \mu \mathrm{M}$

$\mathrm{D}$

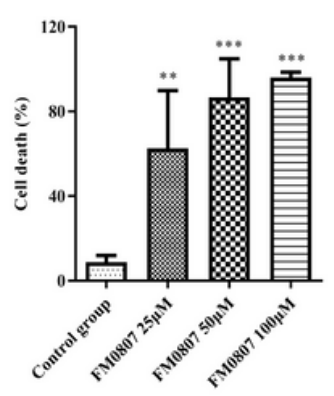

PI
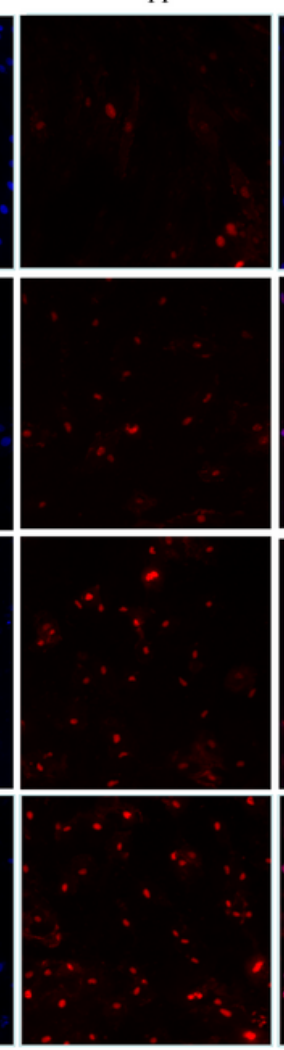

Merge
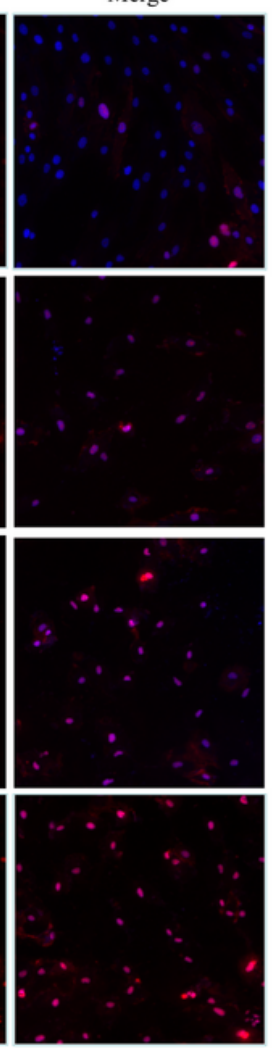

\section{Figure 1}


Effect of FM0807 on proliferation of RA-FLS (A) RA-FLS morphology under the microscope after applying different concentrations of FM0807(20x®;(B) The effect of FM0807 on RA-FLS growth inhibition was measured by MTT assay. RA-FLS were treated with various concentrations of FM0807 for $72 \mathrm{~h}$. FM0807 significantly reduced the growth inhibition of RA-FLS in a concentration-dependent manner. Treatment of cells with $41.38 \mu \mathrm{M}$ FM0807 for $72 \mathrm{~h}$ led to a nearly $50 \%$ decrease in cell growth, which are shown as the result ( $95 \%$ confidence interval). The OD values in $570 \mathrm{~nm}$ were detected by a microplate reader. (C)The effect of FM0807 on death was determined by double staining with Hoechst 33342/propidium iodide (PI) and High Content Screening. Representative images for each drug treatment. Blue-labeled indicates total cells, Red-labeled cells are dead cells, or necrotic cells or late apoptotic cells. (D) PI positive rates of RAFLS were analyzed via High Content Screening after FM0807 treatment for 72 hours, ${ }^{\star}{ }^{2}<<0.01$, $\star \star \star * P<0.001$ vs control group.

Fig 2

A
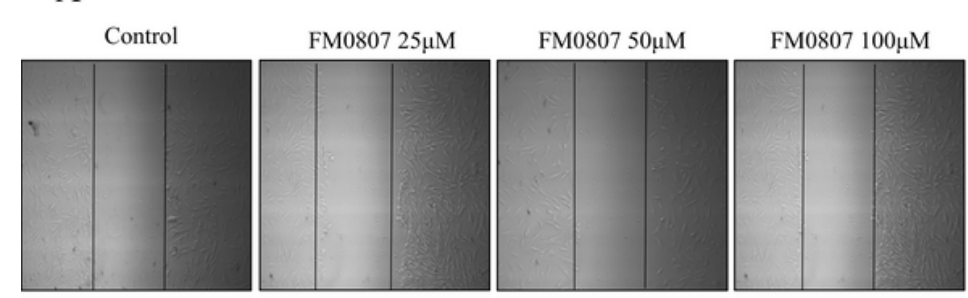

$\mathrm{C}$
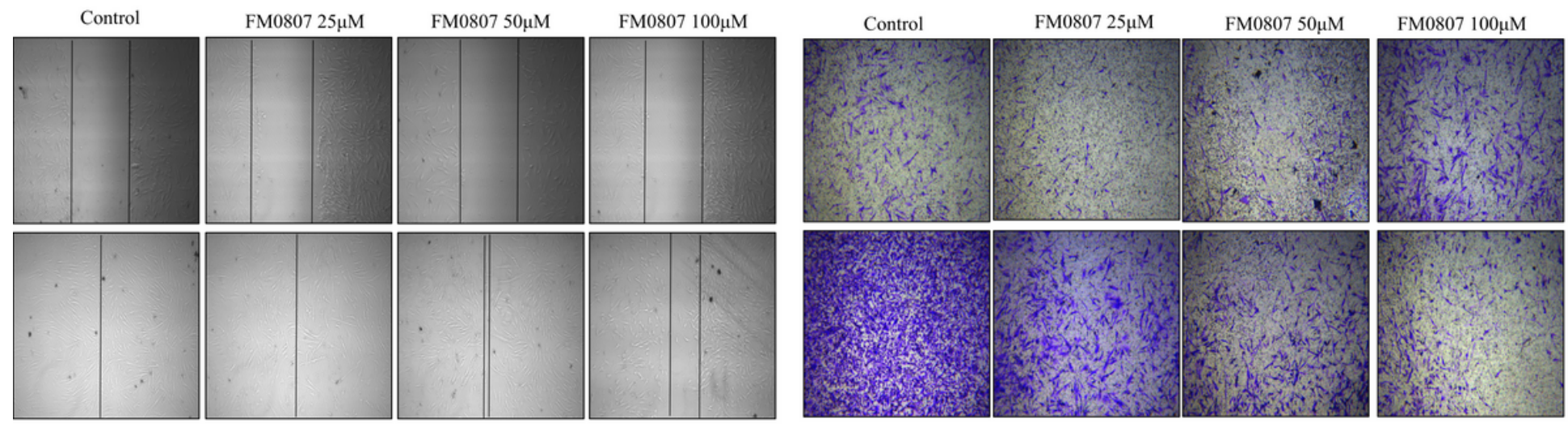

B
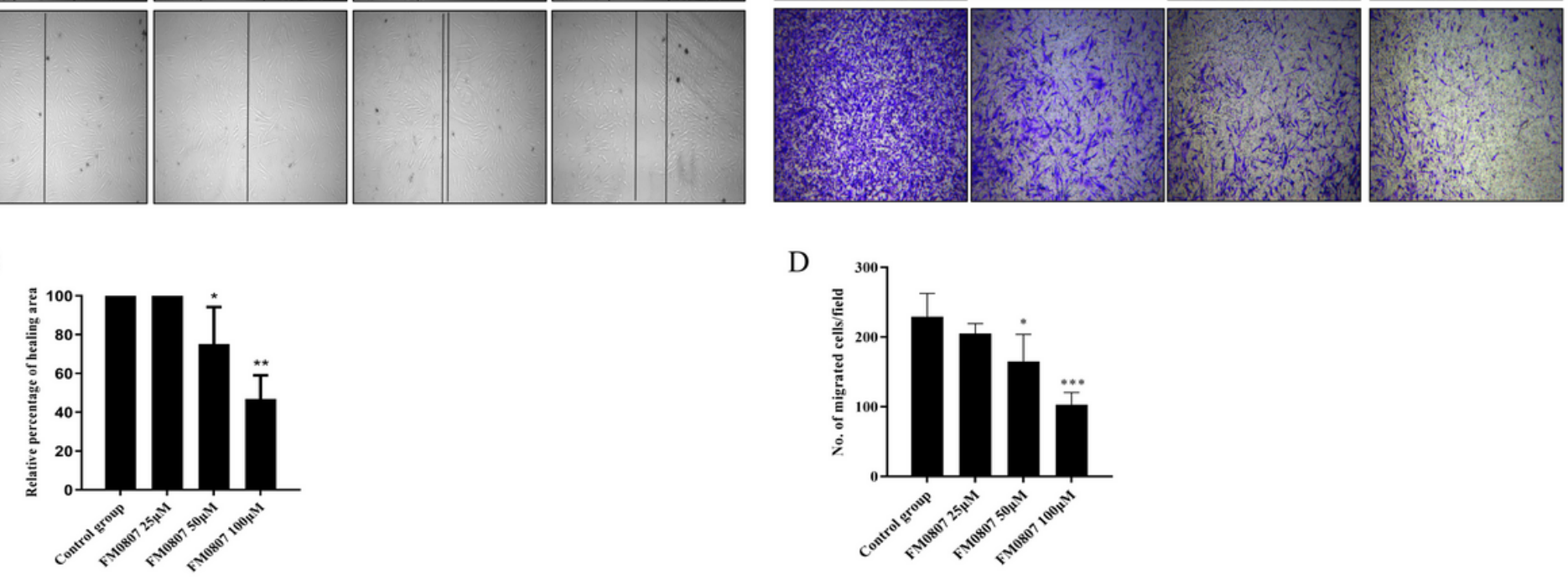

$\mathrm{D}$

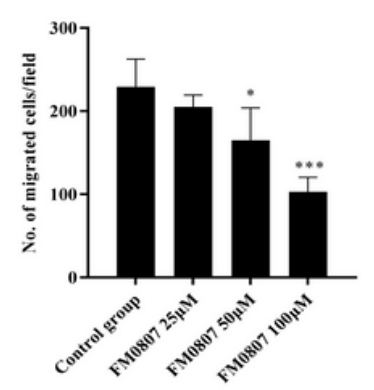

Figure 2

Effect of FM 0807 on the migration ability of RA-FLS in vitro (A) Effect of FM0807 on wound-healing of RA-FLS. (B) Quantitative analysis of scratch closure changes. (C) Effect of FM0807 on Transwell migration viability of RA-FLS. (D) Quantitative analysis of numbers for the migration cell from control and different concentrations FM0807-treated RA-FLS. Experiments were carried out as described in Section 2.4. Data represent mean $\pm S D$ for three independent experiments. Superscript letters a designate a significant differences. ${ }^{\star} P<0.05 \rrbracket^{\star *} P<0.01,{ }^{\star \star *} P<0.001$ vs control group. 


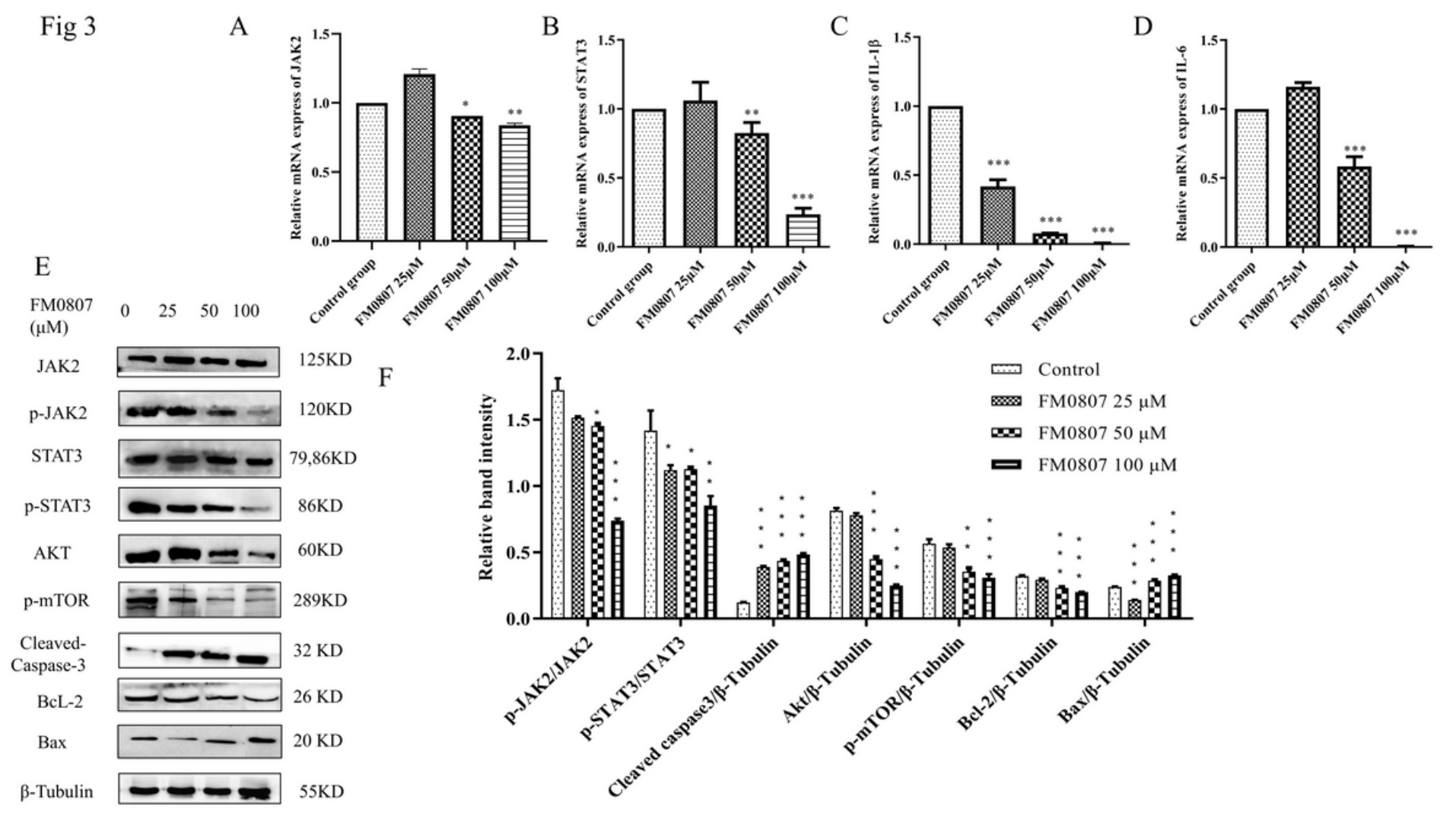

Figure 3

Effect of FM0807 on the expression of JAK2/STAT3 signaling pathway in RA-FLS RA-FLS was stimulated with different concentrations FM0807 $(0 \mu \mathrm{M}, 25 \mu \mathrm{M}, 50 \mu \mathrm{M}, 100 \mu \mathrm{M})$ for $72 \mathrm{~h}$. After culture, the relative mRNA expression was analyzed by RT-PCR. (A)The expression levels of JAK2 mRNA; (B)The expression levels of STAT3 mRNA; (C)The expression levels of IL-1 $\beta$ mRNA; (D)The expression levels of IL-6 mRNA; GADPH mRNA expression was used as control.(E)Representative Western blot analysis of p-JAK2/JAK2, p-STAT3/STAT3, Akt, p-mTOR, caspase-3, Bcl-2 and Bax with the expression of $\beta$-Tubulin protein as an internal control. (F) Quantitative densitometry of p-JAK2/JAK2, p-STAT3/STAT3, Akt, p-mTOR, Cleaved caspase-3, Bax and Bcl-2 leves relative to $\beta$-tubulin . ${ }^{\star} P<0.05$, ${ }^{\star *} P<0.01$ and ${ }^{\star} * \star P<0.001$ vs control group.

\section{Supplementary Files}

This is a list of supplementary files associated with this preprint. Click to download.

- 1.JAK2.tif

- 2.PJAK2FLS.tif

- 3.STAT3.png

- 4.pSTAT3FLS.tif

- Theoriginalimagewesternbolt.docx 